\title{
Efficacy of patterned scan laser in treatment of macular edema and retinal neovascularization
}

This article was published in the following Dove Press journal:

Clinical Ophthalmology

6 August 2009

Number of times this article has been viewed

\section{Dimple Modi \\ Paulpoj Chiranand \\ Levent Akduman}

Saint Louis University School of Medicine, Department of Ophthalmology, Saint Louis University Eye Institute, St. Louis, Missouri, USA
Correspondence: Levent Akduman Department of Ophthalmology, Saint Louis University Eye Institute, 1755 South Grand Blvd., St. Louis, Missouri 63104, USA

$\mathrm{Tel}+$ I 3|4-256-3200

Email lakduman@pol.net
Purpose: To analyze the benefits, efficacy, and complications of the PASCAL ${ }^{\circledR}$ photocoagulation laser system (OptiMedica, Santa Clara, CA, USA) in patients treated at our institution.

Methods: We conducted a retrospective chart review of 19 patients (28 eyes) who underwent laser treatment using the PASCAL ${ }^{\circledR}$ photocoagulation system from November 2006 to November 2007. These 28 eyes were divided into two groups; group 1 eyes underwent macular grid laser and group 2 eyes underwent panretinal photocoagulation. Treatment was performed for macular edema or for iris or retinal neovascularization. Outcomes measured included best-corrected visual acuity (BCVA), efficacy of laser treatment, complications, duration of the procedure, and pain perception, which were noted in the charts for panretinal treatments.

Results: Follow-up was $5.9 \pm 2.6$ months for group 1 and $5.9 \pm 4.0$ months for group 2 . In group 1,9/28 eyes required a second treatment for remaining edema. BCVA was stable or better in $66 \%(14 / 21)$ and average central foveal thickness on ocular coherence tomography improved in $71 \%$ (15/21). Time to completion for a number of laser patterns for grid photocoagulation was felt to be too long for completing the total pattern safely, although we have not noted any related complications. In group 2, the neovascularization regressed at least partially in 3/7 patients. Patient-reported pain perception was 3.6 on a scale of 1 to 10 for group 2. Occasional hemorrhages occurred secondary to irregular laser uptake at different spots in the patterns. We observed no visual outcome consequences because of these hemorrhages during follow-up.

Conclusions: Retinal photocoagulation by the PASCAL ${ }^{\circledR}$ laser has comparable efficacy to historical results with conventional retinal photocoagulation in short-term follow-up. PASCAL ${ }^{\circledR}$ photocoagulation can be performed quicker with less discomfort for patients.

Keywords: diabetic retinopathy, laser, macular edema, PASCAL, patterned scan laser, photocoagulation, retinal neovascularization

\section{Introduction}

Laser photocoagulation in various forms is a standard of care procedure used in many retinal vascular disorders, including diabetic retinopathy and retinal vascular occlusions. Laser can be delivered with different machines and modalities (slit lamp, endolaser, indirect laser) at different wavelengths (532 to $810 \mathrm{~nm}$ ) with varying parameters (power, spot size, duration, number of spots).

Conventional laser machines provide a single spot with the duration of each spot to be $50 \mathrm{~ms}$ or more. Spot size varies based on the treatment given; for example, grid and focal macular treatment spot size ranges from 50 to 100 and panretinal laser photocoagulation (PRP) from 200 to 500. The number of spots to be placed also varies for each condition; often times, macular lasers require 10 to 100 spots in each sitting 
and panretinal lasers require 700 to 1500 spots per treatment by conventional laser.

Macular laser treatments occasionally may be painful and retrobulbar anesthesia may be needed. Pain is associated with positional placement of spots; the patient perceives more pain the more anterior (peripheral) the spots are placed. ${ }^{1}$ Horizontal placement of spots is also more painful since this is the area of passage of the posterior ciliary nerves. ${ }^{1}$ Other parameters causing increased pain include longer duration, larger spot size, previous laser sessions, and higher wavelength. ${ }^{1}$

A macular laser procedure usually takes minutes to complete, while a PRP may take 20 to 30 minutes, based on patient cooperation, surgeon experience, and the size of the treatment area.

New laser delivery methods using retinal tracking systems and predetermined laser patters have been widely studied. Patterned scan laser (PASCAL ${ }^{\circledR}$; OptiMedica Corp., Santa Clara, CA, USA) was introduced in 2006 and uses a technology based on the use of a semi-automated application of $532 \mathrm{~nm}$ laser pulses in a predetermined pattern and rapid sequence. Using this device, 0 to $2000 \mathrm{~mW}$ frequency-doubled $\mathrm{Nd}$ :YAG diode laser energy can be applied at 10 to $1000 \mathrm{~ms}$ pulses with $10-\mathrm{ms}$ intervals. ${ }^{2}$ With this technique, more evenly distributed laser burns can be placed in a shorter period of time. The shorter duration also is suggestive of less pain experienced by patients. Another proposed advantage includes higher precision, especially for macular grid lasers, due to the fixation beam that allows patients to remain steady.

Here, we report our experience in treating diabetic retinopathy and retinal vascular occlusions using the PASCAL ${ }^{\circledR}$ within a 1-year time period.

\section{Material and methods}

During the period November 2006 to November 2007, 19 patients at O'Donnell Eye Institute were treated with PASCAL ${ }^{\circledR}$ photocoagulation (Figure 1). This retrospective

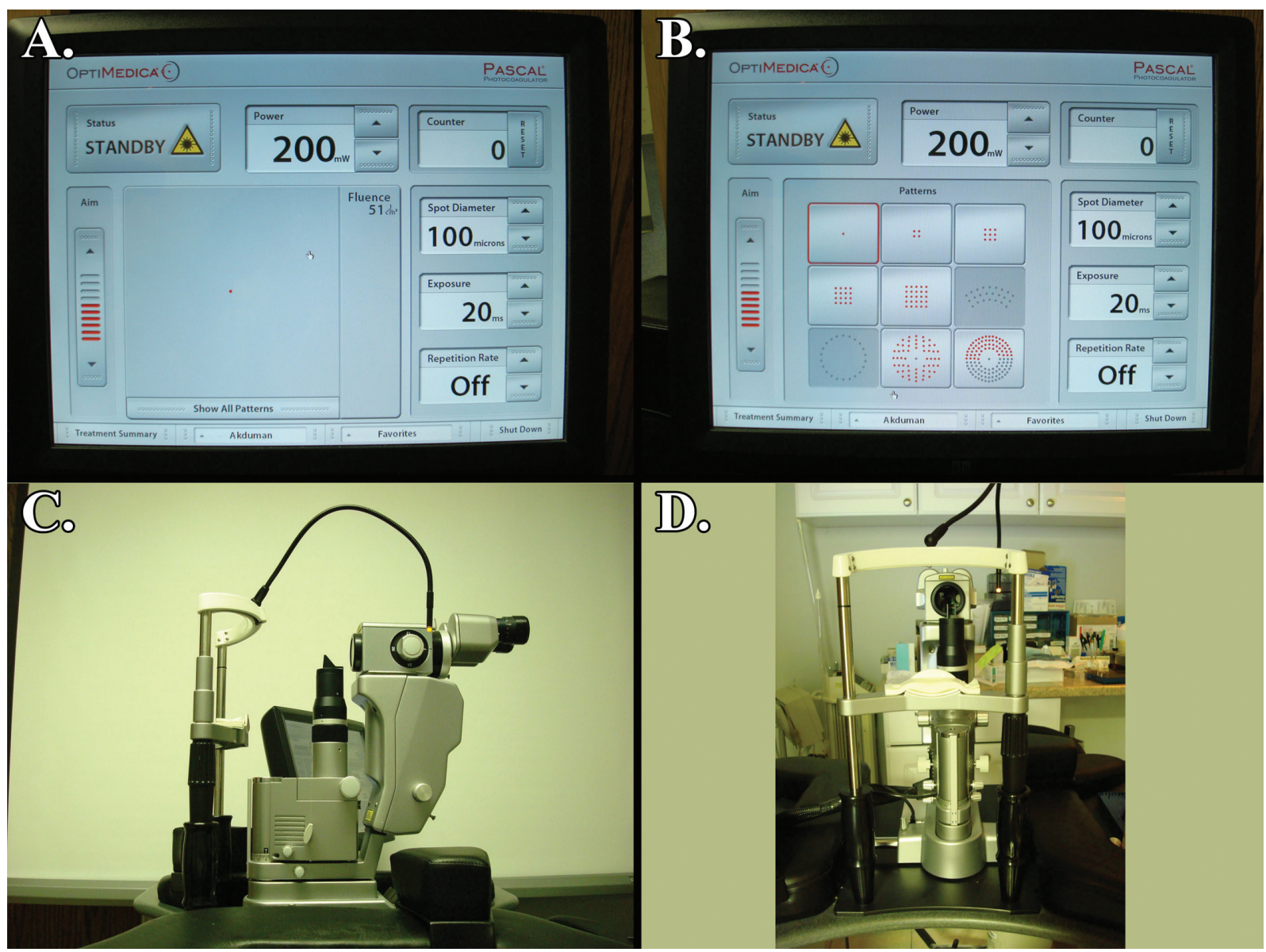

Figure I The PASCAL ${ }^{\circledR}$ system is mounted on a slit lamp. The slit lamp table dimensions are $122 \mathrm{~cm}$ wide by $76 \mathrm{~cm}$ deep by $7 \mathrm{I}$ to $96 \mathrm{~cm}$ high. The height of the table can be easily controlled to adjust for patients' height and body habitus. OptiMedica states that the slit lamp table is also wheelchair accessible. We have found it to be wheelchair accessible, but still does not entirely fit the extremes of patients' height and body habitus. A. Touch-screen used to control PASCAL. B. Pattern options for treatment. C. Side profile of PASCAL. D. Patient's view of PASCAL. 
chart review included 28 eyes that were divided into two groups based on whether they needed treatment for macular edema or for iris or retinal neovascularization secondary to diabetic retinopathy or other ischemic retinal vascular disorders. Macular edema was measured pre- and postoperatively at each visit by Stratus Ocular Coherence Tomography (OCT) (Carl Zeiss Meditec Inc., Dublin, CA, USA).

Our outcome measures were noted by best corrected visual acuity (BCVA), efficacy of laser treatment, duration of the laser treatment, and pain perception. BCVA was converted to $\log$ MAR for statistical analysis and then converted back to Snellen acuity for the ease of interpretation. Efficacy of laser treatment was measured by 1) a decrease in macular edema using OCT if treatment was aimed at macular edema, or 2) regression in neovascularization of the iris or retina if treatment was aimed at ocular ischemic vascular disorders. Patients that received PRP were asked to report their pain perception on a scale of 1 to 10,10 being the most severe. Topical proparacaine was used for local anesthesia prior to performing the procedure.

\section{Results}

Group 1 consisted of 12 patients and 21 eyes. Grid laser was performed in these 21 eyes (Figure 2). Of these, macular edema was secondary to diabetic retinopathy in 18 of the 21 eyes and secondary to branch retinal vein occlusion (BRVO) is 3 of the 21 eyes. The mean pre-laser BCVA was 20/54 and mean post-laser best-corrected visual acuity was 20/56. Average central foveal thickness (CFT) was

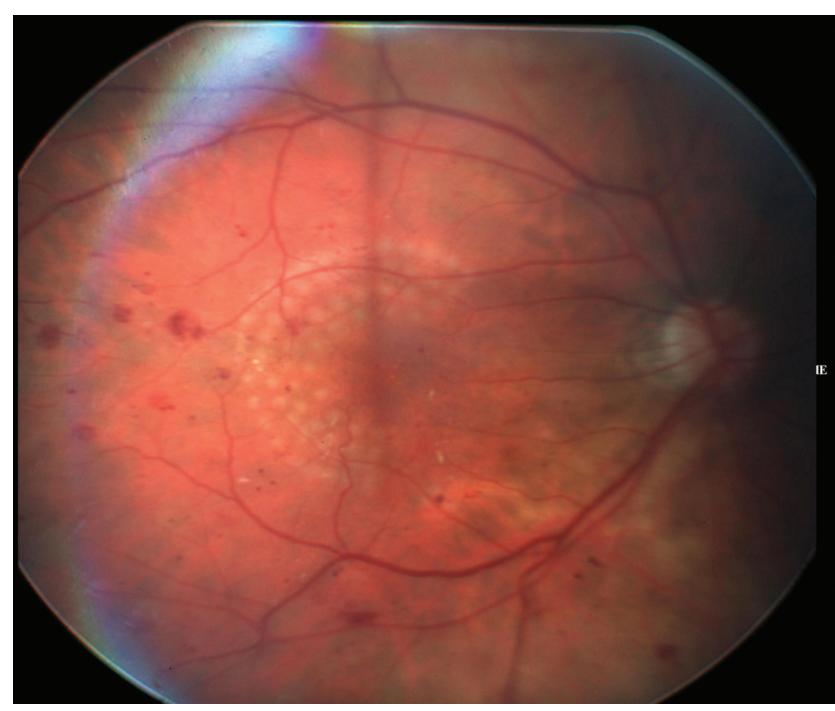

Figure 2 Grid laser with PASCAL ${ }^{\circledR}$.
Table I Treatment of macular edema with PASCAL ${ }^{\circledR}$

\begin{tabular}{lll}
\hline Type of laser & $\begin{array}{l}\text { Focal grid } \\
\text { laser }\end{array}$ & $\begin{array}{l}\text { Panretinal } \\
\text { photocoagulation }\end{array}$ \\
\hline Number of patients & 12 & 7 \\
Number of eyes & 21 & 7 \\
Average pre-laser BCVA & $20 / 54$ & $20 / 94$ \\
Average post-laser BCVA & $20 / 56$ & $20 / 97$ \\
Average pain & N/A & 3.6 \\
Number of eyes with & 2 & 2 \\
complications $^{\mathrm{a}}$ & & \\
\hline
\end{tabular}

aComplications include retinal hemorrhages.

Abbreviations: BCVA, best-corrected visual acuity.

used as the main indicator of increased macular edema or decreased macular edema. CFT improved in $71 \%(15 / 21)$ eyes (Table 1).

Group 2 consisted of seven patients (seven eyes) receiving panretinal photocoagulation during a single session because of neovascularization of the retina or iris secondary to ischemic retinal disorders (Figure 3). Six of the seven patients received PRP because of proliferative diabetic retinopathy and one of the seven patients received PRP because of neovascularization from a past history of central retinal vein occlusion (CRVO). For these patients, pre-laser BCVA was 20/94 and post-laser BCVA was 20/97. Neovascularization at least partially regressed in three of the seven patients.

In group 2, five of the seven patients were asked to rate procedural pain. The average pain felt by these five patients averaged 3.6 using only topical proparacaine as anesthesia. The average number of spots used was 1326 , the maximum

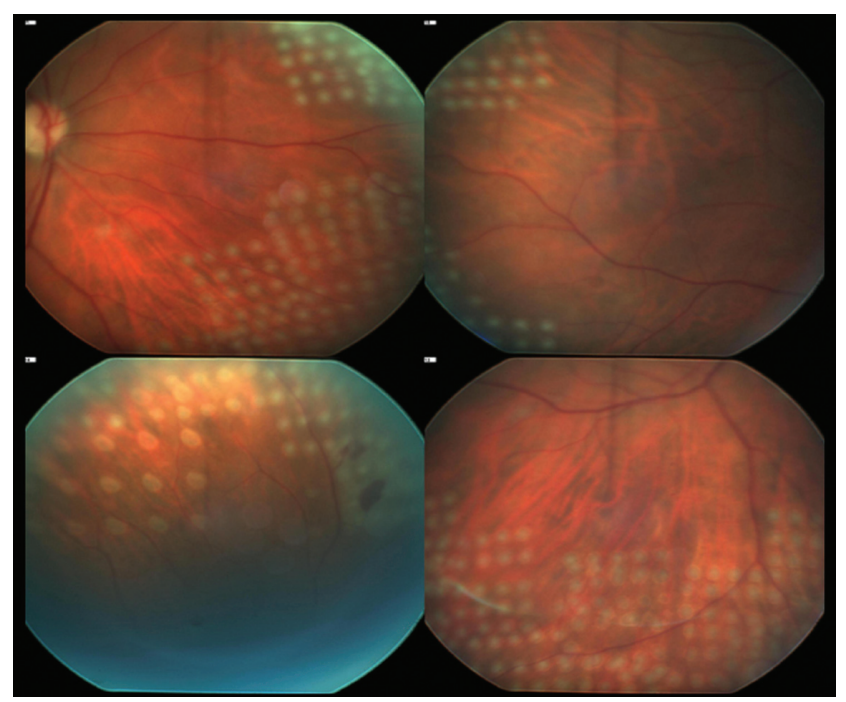

Figure 3 Panretinal photocoagulation. 
number used being 1825. All PRP sessions took less than 5 minutes of laser time.

Of the 28 eyes examined, there were two complications in our patients; retinal hemorrhage occurred secondary to too high of a power being used within the pattern (Figure 4). These minor hemorrhages did not, however, reflect upon the patients' visual acuity in our study. No new choroidal neovascularization occurred from these intense burns. Inconsistencies in uptake during PRP occurred when different patterns were used.

\section{Discussion and conclusion}

Laser photocoagulation remains the gold standard in the treatment of many retinal vascular disorders. Conventional photocoagulation uses a single application of laser energy per laser shot. PASCAL ${ }^{\circledR}$ system uses a multitude of patterns including square arrays, single arc, and full and partial macular grid, although also capable of single spot laser. Pan-retinal photocoagulation for proliferative diabetic retinopathy and macular grid for diabetic macular edema are the primary applications; however, patterns can be applied for other retinal vascular disorders and retinal tears.

Sanghvi et al describe panretinal photocoagulation done with conventional one-spot laser versus the multiple-spot pattern laser done with the PASCAL ${ }^{\circledR}{ }^{2}$ Twenty-two patients had both types of methods used in the same eye. The mean power was $235 \mathrm{~mW}$ for the conventional laser method

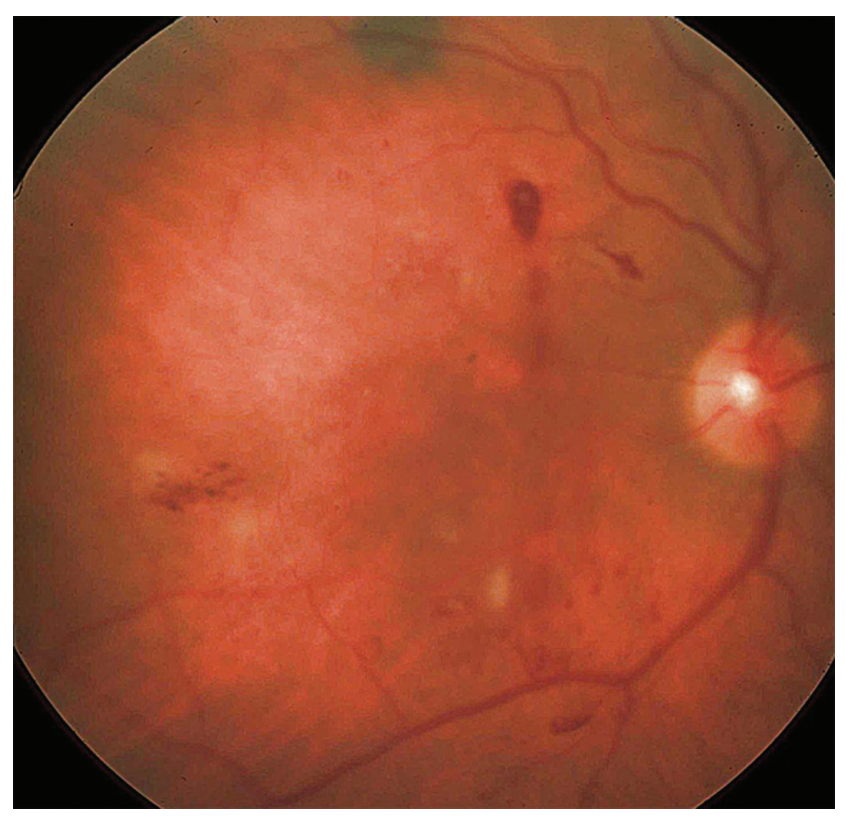

Figure 4 Retinal hemorrhage and leak into the vitreous from a single laser spot in the superior aspect of the retina where grid laser was performed. versus $396 \mathrm{~mW}$ for the $\mathrm{PASCAL}^{\circledR}$ to achieve the same type of laser burn. ${ }^{2}$ This was thought to be due to a much shorter duration of laser exposure time used by the PASCAL ${ }^{\circledR}$. Also, when comparing PRP done by conventional laser versus PASCAL ${ }^{\circledR}$ on the same eye, the patient received many more laser spots in the PASCAL ${ }^{\circledR}$ group, with a mean of 738 to 1116 spots respectively. ${ }^{2}$ This was thought to be a result of better toleratance of the laser spots in the PASCAL ${ }^{\circledR}$ group because of the shorter exposure time of the laser. Similarly, in patients who had undergone laser treatment for macular edema, the PASCAL ${ }^{\circledR}$ group used more power, again due to a shorter exposure time of the laser. However, the power levels required with the PASCAL ${ }^{\circledR}$ system did not result in any complications in that study. In our experience performing grid and PRP with the PASCAL ${ }^{\circledR}$ we realized that retinal hemorrhages were common because of irregular uptake. Yet, this resulted in no significant complications other than a few days of floaters voiced by the patients. ${ }^{3}$

Blumenkranz et al noted that the power required to produce ophthalmoscopically visible spots decreased with increasing pulse duration. ${ }^{4}$ In their experiments, cumulative pulse energy increased with pulse duration, indicating a significant diffusion of heat from the laser spot with pulse durations greater than $20 \mathrm{~ms}$, which is typically longer than the duration used in the PASCAL ${ }^{\circledR}$ system. Also noted was creation of less homogenized and less localized lesions with longer pulses. At $20 \mathrm{~ms}$ pulse duration, the threshold power for producing a visible lesion was $110 \mathrm{~mW}$ to $120 \mathrm{~mW}$ and the threshold for hemorrhage was $600 \mathrm{~mW}^{4}$ Blumenkranz et al also estimated the time to place 16 laser spots at one application and the time to place 36 spots of laser at one application with pulse durations of $10 \mathrm{~ms}$, $20 \mathrm{~ms}, 50 \mathrm{~ms}$, and $100 \mathrm{~ms}^{4}$ They estimated that it may be possible to reduce total laser application time by a factor of 7- to 10-fold using these pattern arrays rather than single spot photocoagulation. With these data, a PRP can potentially be done in one sitting rather than multiple sittings with the conventional laser method. Potential advantages over conventional single-spot photocoagulation include improved efficiency, increased uniformity and precision of spot placement, and possibly reduced pain and visual field defects because of reduced heat diffusion toward the choroid and inner retina. ${ }^{3}$

However, in the DRS and ETDRS, recommendations are that no more than 900 applications per session be used, separated in time by 2 weeks because of the potential for complications such as macular edema, choroidal detachment, exudative retinal detachment, and shallowing or closure of the 
anterior chamber angle. ${ }^{5,6}$ Single versus multiple treatment sessions using argon laser for PRP caused by proliferative diabetic retinopathy was done and visual acuity was assessed after PRP in both the multiple- and single-treatment groups; no difference between both groups was seen in final visual acuity. ${ }^{6}$ Prior to treatment, all patients had high risk for severe visual loss with three of four retinopathy risk factors. ${ }^{2}$ Six months after treatment, only $40 \%$ of the single treatment group versus $36 \%$ of the multiple treatment group was in the same high-risk category. ${ }^{7}$ When compared, the single- and multiple-treatment groups were of equal efficacy in reducing the number of retinopathy risk factors. ${ }^{7}$ Also, in this study there did not appear to be a marked difference between single- and multiple-treatment session groups in regression of neovascularization of the optic disc. A higher incidence of exudative retinal detachment, choroidal detachment, and angle closure occurred in the single treatment group eyes than in the multiple treatment group eyes. ${ }^{8}$ All of these complications resolved spontaneously within a short period of time after treatment and visual loss that was acquired from these complications were only transient. ${ }^{8}$

Jain et al demonstrated that shorter pulse durations decreased the width and axial extent of the retinal lesions. ${ }^{9}$ There was less collateral damage with less spread of heat to adjacent retina and choroid. The PASCAL ${ }^{\circledR}$ system uses durations in this range, and with the less spread of heat to the adjacent retina and choroid with the shorter duration, even one sitting PRP sessions may not have significant transient choroidal or retinal detachments. Clinical efficacy in the long term remains to be studied.

Al-Hussainy et al used an ND:YAG laser with a wavelength of $532 \mathrm{~nm}$ with shorter duration exposures to determine if this was more comfortable for patients than conventional parameters. ${ }^{1}$ Their study compared exposure times of 0.1 seconds with a duration of 0.02 seconds. The group that had a longer exposure time required less power averaging $0.178 \mathrm{~W}$ compared to the shorter duration group needing $0.49 \mathrm{~W}^{1}{ }^{1}$ These patients were asked to rate their pain perception on a scale of 0 to 10 with 0 being no pain and 10 being the most pain they have ever experienced. The patients with the longer exposure time rated the pain on average to be 5.11. The patients with the shorter duration time rated the pain to be on average 1.41 . This was found to be statistically significant. ${ }^{9}$ The PASCAL ${ }^{\circledR}$ system uses the same laser and uses this shorter duration of action. The mode of delivery that the PASCAL ${ }^{\circledR}$ system uses looks highly promising in decreasing patient pain during PRP. In our study, $90 \%$ of the patients reported a pain perception
3.6 with a range from 2 to 9 using a pain scale from 1 to 10 , 10 being the most severe pain. ${ }^{3}$

As we have discussed previously, laser-tissue interaction is influenced by wavelength, spots size, power, and exposure time. According to our experiences as well as other studies, a higher power is required to produce the desired lesion on the retina. Sanghvi et al did not observe any complications. ${ }^{2}$ However, retinal hemorrhages from macular photocoagulation and PRP due to irregular uptake from differing amounts of pigmentation of the retina, differences in media clarity, and differences in the focusing distance among the spots occurred. Yet, this resulted in no significant complications other than a few days of floaters voiced by the patients. ${ }^{2}$ Laser complications such as this needs to be meticulously investigated further to reduce such complications. Some of the pattern and parameter alternatives readily presented currently in the software need to be modified for the safest applications. ${ }^{10}$

PASCAL $^{\circledR}$ photocoagulation can be performed quicker with less discomfort for the patients. Follow-up for this study was less than a year for both groups. The results of both of these groups show equal efficacy to traditional 1-spot laser. However, further long-term follow-up needs to be done. Also, only 28 eyes were used in this group. A larger study needs to be done in the future to better understand further the benefits and disadvantages of using this system.

\section{Acknowlegments and disclosures}

This study was supported in part by a departmental fund from Research to Prevent Blindness (RPB). The authors disclose no conflicts of interest.

\section{References}

1. Al-Hussainy S, Dodson PM, Gibson JM. Pain response and followup of patients undergoing panretinal laser photocoagulation with reduced exposure times. Eye. 2008;22:96-99.

2. Sanghvi C, McLauchlan R, Delgado C, et al. Initial experience with the PASCAL ${ }^{\circledR}$ photocoagulator: a pilot study of 75 procedures. $\mathrm{Br} J$ Ophthalmol. 2008;92:1061-1064.

3. Chiranand P, Akduman L. Efficacy of PASCAL ${ }^{\circledR}$ Photocoagulation in treatment of retinal disorders. World Ophthalmology Congress. Hong Kong. June 2008.

4. Blumenkranz MS, Yellachich D, Andersen DE, et al. Semiautomated patterned scanning laser for retinal photocoagulation. Retina. 2006;26:370-376.

5. The Early Treatment Diabetic Retinopathy Study Research Group. Techniques for scatter and local photocoagulation treatment of diabetic retinopathy: Early Treatment Diabetic Retinopathy Study report no. 3. Int Ophthalmol Clin. 1987;27:254-264.

6. Early Treatment Diabetic Retinopathy Study Research Group. Treatment techniques and clinical guidelines for photocoagulation of diabetic macular edema. Early Treatment Diabetic Retinopathy Study report number 2. Ophthalmology. 1987;94: 761-774. 
7. Doft BH, Blankenship GW. Single versus multiple treatment sessions of argon laser panretinal photocoagulation for proliferative diabetic retinopathy. Ophthalmology. 1982;89:772-779.

8. Diabetic Retinopathy Study Research Group. Four risk factors for severe visual loss in diabetic retinopathy: the third report from the diabetic retinopathy study. Arch Ophthalmol. 1979;97:654-655.
9. Jain A, Blumenkranz MS, Paulus Y, et al. Effect of pulse duration on size and character of the lesion in retinal photocoagulation. Arch Ophthalmol. 2008;126:78-85.

10. Bloom SM, Brucker AJ. Laser Surgery of the Posterior Segment. 2nd ed. Lippincott-Raven: Philadelphia; 1997.

\section{Publish your work in this journal}

Clinical Ophthalmology is an international, peer-reviewed journal covering all subspecialties within ophthalmology. Key topics include: Optometry; Visual science; Pharmacology and drug therapy in eye diseases; Basic Sciences; Primary and Secondary eye care; Patient Safety and Quality of Care Improvements. This journal is indexed on

\section{Dovepress}

PubMed Central and CAS, and is the official journal of The Society of Clinical Ophthalmology (SCO). The manuscript management system is completely online and includes a very quick and fair peer-review system, which is all easy to use. Visit http://www.dovepress.com/ testimonials.php to read real quotes from published authors. 